\title{
Orthostatic hypotension and age-related sarcopenia
}

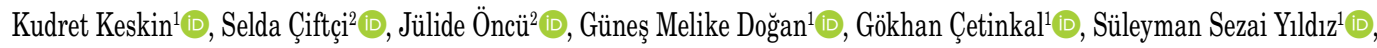 \\ Serhat Sığırc1iD, Kadriye Orta Kılıçkesmez ${ }^{1}$ (D) \\ ${ }^{1}$ Department of Cardiology, University of Health Sciences, Şşli Hamidiye Etfal Training and Research Hospital, Istanbul, Turkey \\ ${ }^{2}$ Department of Physical Medicine and Rehabilitation, University of Health Sciences, Şişli Hamidiye Etfal Training and Research Hospital, Istanbul, Turkey
}

Received: October 23, 2019 Accepted: January 09, 2020 Published online: March 04, 2021

\begin{abstract}
Objectives: This study aims to determine the association of sarcopenia with orthostatic hypotension $(\mathrm{OH})$ which is a significant precursor to falls and related injuries in elderly patients.

Patients and methods: A total of 91 outpatients (18 males, 73 females; mean age $79.3 \pm 4.0$ years; range, 75 to 91 years) were prospectively enrolled and those who were eligible underwent comprehensive sarcopenia assessment including measurement of muscle mass, strength, physical performance, anthropometric measurements along with frailty tests. Patients classified as sarcopenic or non-sarcopenic based on these measurements underwent supine and standing blood pressure (BP) measurements. The frequency of $\mathrm{OH}$ was compared between the two groups.

Results: Of the 91 patients, 29 (31.9\%) had sarcopenia. There was no statistical difference in measurements of functional tests which consisted of gait speed, timed up-and-go test and handgrip strength. However, timed sit-to-stand test values were higher in sarcopenic patients $(18.2 \pm 7.9$ vs. $15.0 \pm 5.1, \mathrm{p}=0.04)$. Patients with sarcopenia developed OA and intolerance more often compared to the non-sarcopenic patients $(n=15[50.0 \%]$ vs. $n=14$ [23.0\%], $p<0.01$ and $n=13[44.8 \%]$ vs. $n=9[15.3 \%], p<0.01$, respectively). The adjusted odds ratio for sarcopenia was 7.80 (95\% confidence interval 1.77-34.45), $\mathrm{p}=0.007$.
\end{abstract}

Conclusion: Age-related sarcopenia increases the risk of OA in the elderly. This may in part explain the increased incidence of falls and also help identification of risky elderly patients for orthostatic BP drops.

Keywords: Blood pressure, elderly, frailty, orthostatic hypotension, sarcopenia.

Sarcopenia is a syndrome that refers to the progressive loss of skeletal muscle mass along with low muscle strength and physical performance. ${ }^{[1]}$ There is mounting evidence showing that sarcopenia is associated with many negative outcomes, such as falls, functional disability, poor quality of life and death. ${ }^{[2,3]}$ Although age-related sarcopenia of the elderly people has been observed for a long time, the increased incidence of falls and postural problems these patients encounter are generally attributed to gait problems, dizziness, low physical performance and frailty without much emphasis on the underlying other etiologies. ${ }^{[4,5]}$
However, sarcopenia is a complex syndrome that has significant associations with the cardiovascular and nervous system. ${ }^{[6]}$ Increased arterial stiffness, endothelial dysfunction, systolic hypertension and autonomic nervous system dysfunction are among the pathologies reported so far..$^{[7,8]}$

In this context, orthostatic hypotension $(\mathrm{OH})$, which is commonly seen in elderly people and a significant risk factor for cardiovascular events, has not been investigated in elderly patients with sarcopenia. By definition, $\mathrm{OH}$ is a chronic autonomic cardiovascular dysfunction that is characterized

\section{Corresponding author: Kudret Keskin, MD. SBÜ Şişli Hamidiye Etfal Eğitim ve Araştırma Hastanesi Kardiyoloji Kliniği, 34371 Şişli, İstanbul, Türkiye. e-mail: keskinkudret@yahoo.com


by inability to sustain adequate cardiac output to assuming an upright position. Normally, in response to the venous pooling immediately after standing, a number of compensatory mechanisms such as increased venous tone and heart rate should come into play and normalize the blood pressure (BP) and thus the cerebral perfusion. Elderly patients are particularly prone to $\mathrm{OH}$ and related symptoms due to several comorbidities. Since lower extremity muscle contraction is also a significant contributor for the venous blood to return to the heart, sarcopenic elderly patients owing to their low muscle strength along with impaired cardiovascular and nervous system functions may be prone to $\mathrm{OH}$. Based on this, in this study, we aimed to determine the association of sarcopenia with $\mathrm{OH}$ which is a significant precursor to falls and related injuries in elderly patients.

\section{PATIENTS AND METHODS}

This prospective observational study included 91 patients (18 males, 73 females; mean age: $79.3 \pm 4.0$ years; range, 75 to 91 years) referred to Şişli Hamidiye Etfal Training and Research Hospital Cardiology and Osteoporosis Outpatient Departments. In order to reduce the confounding factors for development of $\mathrm{OH}$ and to ensure the accuracy of bioimpedance muscle mass measurements, patients with neurodegenerative diseases, end-stage renal disease, malignancy, heart failure, significant dehydration, anemia (hemoglobin $<8 \mathrm{~g} / \mathrm{dL}$ ), acute infection, edema, pace-maker and those who could not stand up were excluded. In addition, all participants underwent transthoracic echocardiography to rule out heart failure and major cardiac disorders that might have an impact on BP measurements. Based on sarcopenia-related measurements, patients were divided into two groups (sarcopenic vs. non-sarcopenic). The study protocol was approved by the Şişli Hamidiye Etfal Training and Research Hospital Ethics Committee (ClinicalTrials.gov Identifier Number: 03732716). A written informed consent was obtained from each patient. The study was conducted in accordance with the principles of the Declaration of Helsinki.

Sarcopenia was defined according to the European Working Group on Sarcopenia in Older People (EWGSOP) criteria in which low muscle mass plus either low muscle strength or physical performance were required for diagnosis. ${ }^{[1]}$ When assessing muscle mass, we used Tanita-305
(Tanita Corp., Tokyo, Japan) multi-frequency bioelectrical impedance body composition analyzer. All measurements were performed in the morning when patients were fasting for $8 \mathrm{~h}$ and with an empty bladder. Based on the EWGSOP criteria, skeletal muscle mass indexes less than $8.87 \mathrm{~kg} / \mathrm{m}^{2}$ for males and $6.42 \mathrm{~kg} / \mathrm{m}^{2}$ for females were defined as cut-off values for sarcopenia. Skeletal muscle mass index was calculated as appendicular mass in kilograms divided by the square of height in meters.

Muscle strength was assessed by measuring handgrip strength using a Jamar dynamometer (Sammons Preston Rolyan, Chicago, USA). The test was performed while the patient was seated with shoulder adducted and forearm neutrally rotated, elbow flexed to 90 degrees, and forearm in a neutral position. After performing three consecutive contractions with the dominant arm, the highest value was used for analysis. Handgrip strengths less than $30 \mathrm{~kg}$ for males and $20 \mathrm{~kg}$ for females were defined as low muscle strength based on EWGSOP criteria.

Physical performance was assessed using the timed up-and-go (TUG) test in which a patient was instructed to stand from a sitting position, quickly walk three meters and walk back to the chair and sit down again. The duration of the test was reported in seconds. Generally, a score of more than $15 \mathrm{sec}$ portend poor performance. Gait speed was derived from the TUG test using the following formula: $\left[6 /(\text { TUG time) }]^{*} 1.62\right.$. Gait speed less than $0.8 \mathrm{~m} / \mathrm{sec}$ was defined as the cut-off value for definition of low physical performance. The four-item essential frailty toolset (EFT) which was previously validated in a large cohort was deemed appropriate for frailty assessment. ${ }^{[9]}$ It incorporated four different measurements which were timed sit-to-stand (TSTS) test (5 times), mini-mental state examination, serum hemoglobin and albumin levels. The test score ranged from 0 to 5 . EFT score 3 or greater was defined as frailty. The TSTS test measured the time required to stand five times from a seated position without using arms.

All BP measurements were performed in the morning after 8 -h fasting. Patients were taken to a quiet room where supine BP recordings were made following a 5 -min resting period. Three more BP measurements were taken when the patients stood up. These were immediate, $1^{\text {st }}$ and the $3^{\text {rd }}$ min of standing, respectively. All measurements were performed using a fully automated Omron 907XL sphygmomanometer 
(Omron Healthcare Co, Ltd, Kyoto, Japan) that was attached to the patients' upper arm circumference. According to the diagnostic criteria established by the Consensus Committee of the American Autonomic Society and American Academy of Neurology, $\mathrm{OH}$ is defined as a decrease in systolic $\mathrm{BP}$ of $20 \mathrm{mmHg}$ or a decrease in diastolic BP of $10 \mathrm{mmHg}$ within $3^{\text {rd }}$ min of standing. ${ }^{[10]}$ The accompanying symptoms such as dizziness, inability to maintain balance and blurred vision were noted and defined as orthostatic intolerance.

\section{Statistical analysis}

Since $\mathrm{OH}$ is quite prevalent in elderly patients, we anticipated approximately $10 \% \mathrm{OH}$ rate in the control and a three-fold higher rate in the study group. Based on this assumption, each group required 40 patients to reach $80 \%$ power with an alpha level of 0.05 . Continuous variables were expressed as mean \pm standard deviation, or median (interquartile range) and categorical variables were presented as numbers and percentages. The Kolmogorov-Smirnov test was used to assess the distribution pattern of continuous variables. If normally distributed, continuous variables were compared between groups using independentsamples t-test and if the distribution of variables were skewed (log-normal), Mann-Whitney U test was used instead. Categorical data were compared using the chi-square or Fisher exact test. Logistic regression analysis was carried out to measure the risk of having $\mathrm{OH}$ in patients with sarcopenia. Univariate and multivariate logistic regression analysis were performed to assess the association of sarcopenia with $\mathrm{OH}$. Variables that were statistically significant in univariate analysis and of clinical importance were entered into the model without stepwise selection. Model-fit was assessed using the likelihood and $\mathrm{R}$ square values. A $p$ value of $<0.05$ was considered statistically significant. The statistical analysis was performed using IBM SPSS Statistics for Windows, version 20.0 (IBM Corp., Armonk, NY, USA).

\section{RESULTS}

Of the 91 patients, 29 (31.9\%) had sarcopenia. Baseline clinical characteristics and laboratory values are presented in Table 1. Body mass index (BMI) was significantly lower in sarcopenic patients $(23.9 \pm 4.1 \mathrm{vs}$. $29.9 \pm 4.5, \mathrm{p}<0.01)$. There was no statistical difference in terms of chronic diseases such as diabetes mellitus, hypertension and cardiovascular disease. Also, the intensity of antihypertensive treatment $(1.4 \pm 1.2$ vs. $1.7 \pm 1.2, \mathrm{p}=0.28)$ and diuretic use $(\mathrm{n}=7$ [24.1\%] vs. $\mathrm{n}=21$ [33.9\%], $\mathrm{p}=0.34$ ) were similar in both groups. In terms of laboratory values, vitamin $\mathrm{D}$ levels were significantly lower in sarcopenic patients $(27.6 \pm 12.2 \mathrm{vs}$. $34.7 \pm 18.7, \mathrm{p}=0.02$ ).

Measurements related to sarcopenia are presented in Table 2. Skeletal muscle mass index was significantly lower in sarcopenic patients $(6.6 \pm 0.8$ vs. $7.7 \pm 0.9, \mathrm{p}<0.01)$. In addition, all anthropometric measurements including mid-arm and calf were lower in patients with sarcopenia ( $\mathrm{p}<0.01$ for all). However, there was no statistical difference in measurements of functional tests which consisted of gait speed, TUG test and handgrip strength $(0.8 \pm 0.2 \mathrm{sec}$ vs. $0.7 \pm 0.1$ sec, $\mathrm{p}=0.1 ; 13.2 \pm 5.8$ sec vs. $14.2 \pm 4.1 \mathrm{sec}, \mathrm{p}=0.39$; $18.1 \pm 5.3 \mathrm{~kg}$ vs. $18.0 \pm 4.5 \mathrm{~kg}, \mathrm{p}=0.99$, respectively). The only statistical difference was found in TSTS test in which patients with sarcopenia had higher values $(18.2 \pm 7.9 \mathrm{sec}$ vs. $15.0 \pm 5.1 \mathrm{sec}, \mathrm{p}=0.04)$. According to EFT assessment, patients with sarcopenia had lower scores compared to non-sarcopenic patients $(1.4 \pm 1.0$ vs. $1.7 \pm 0.9, \mathrm{p}=0.02)$.

The main outcomes of the study are presented in Table 2. Patients with sarcopenia developed $\mathrm{OH}$ and intolerance more often compared to the nonsarcopenic patients $(n=15$ [50.0\%] vs. $n=14$ [23.0\%], $\mathrm{p}<0.01$ and $\mathrm{n}=13$ [44.8\%] vs. $\mathrm{n}=9$ [15.3\%], $\mathrm{p}<0.01$, respectively). Mean heart rate increased in both groups when standing; (supine: $72.8 \pm 11.4$; standing [immediate]: $80.9 \pm 12.9$; standing [ $1^{\text {st }} \mathrm{min}$ ]: $79.6 \pm 12.1$; standing [ $\left.3^{\text {rd }} \mathrm{min}\right]: 79.7 \pm 12.7$, respectively, in the entire group). The greatest pressure drop was observed immediately after standing. Mean systolic and diastolic BPs are presented in Figures 1 and 2. No cases of $\mathrm{OH}$ based on isolated diastolic pressure drop were observed.

In order to assess the risk of having $\mathrm{OH}$ in sarcopenic patients, a univariate logistic regression analysis was performed in which the odds ratio (OR) was 3.3 (95\% confidence interval [CI]: 1.2-8.5, $\mathrm{p}=0.01$ ). Multivariate analysis was performed including sex, age, BMI and vitamin D levels as covariates. Sarcopenia was still the only independent predictor of $\mathrm{OH}$ development (OR: 7.80 [95\% CI: 1.77-34.45], $\mathrm{p}=0.007)$. Variables were entered into model without stepwise selection method and model fit was assessed according to Nagelkerke R2 and $\log$ likelihood values which were 0.23 and 80.42 , respectively. 


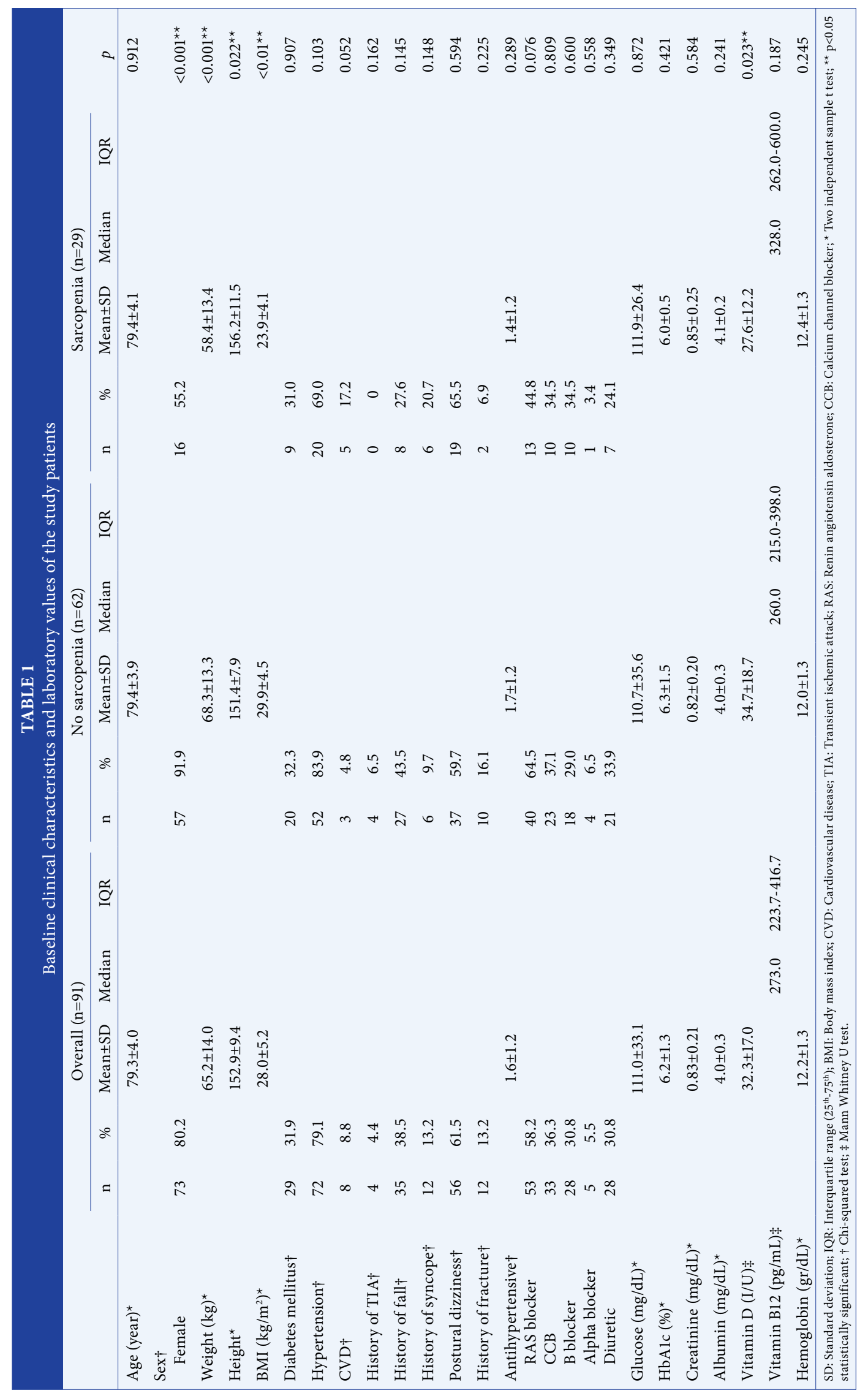




\begin{tabular}{|c|c|c|c|c|c|c|c|c|c|c|}
\hline \multicolumn{11}{|c|}{$\begin{array}{r}\text { TABLE } 2 \\
\text { Sarcopenia related measurements, frailty and the } \mathrm{d}\end{array}$} \\
\hline & \multicolumn{3}{|c|}{ Overall $(n=91)$} & \multicolumn{3}{|c|}{ No sarcopenia $(n=62)$} & \multicolumn{3}{|c|}{ Sarcopenia $(n=29)$} & \multirow[b]{2}{*}{$p$} \\
\hline & $\mathrm{n}$ & $\%$ & Mean \pm SD & $\mathrm{n}$ & $\%$ & Mean \pm SD & $\mathrm{n}$ & $\%$ & Mean \pm SD & \\
\hline Appendicular skeletal muscle mass (kg) & & & $17.4 \pm 3.5$ & & & $18.0 \pm 3.3$ & & & $16.4 \pm 3.8$ & 0.062 \\
\hline Skeletal muscle mass index $\left(\mathrm{kg} / \mathrm{m}^{2}\right)$ & & & $7.4 \pm 1.0$ & & & $7.7 \pm 0.9$ & & & $6.6 \pm 0.8$ & $<0.001^{*}$ \\
\hline \multicolumn{11}{|l|}{ Calf circumference $(\mathrm{cm})$} \\
\hline Right & & & $34.6 \pm 4.4$ & & & $35.9 \pm 4.1$ & & & $31.9 \pm 3.7$ & $<0.001^{*}$ \\
\hline Left & & & $34.4 \pm 4.3$ & & & $35.5 \pm 4.2$ & & & $32.1 \pm 3.7$ & $<0.001^{*}$ \\
\hline \multicolumn{11}{|l|}{ Mid-upper arm circumference $(\mathrm{cm})$} \\
\hline Right & & & $28.4 \pm 4.1$ & & & $30.0 \pm 3.7$ & & & $25.4 \pm 3.2$ & $<0.001^{*}$ \\
\hline Left & & & $28.0 \pm 4.4$ & & & $29.6 \pm 4.1$ & & & $24.8 \pm 3.2$ & $<0.001^{*}$ \\
\hline Gait speed (m/sec) & & & $0.7 \pm 0.2$ & & & $0.7 \pm 0.1$ & & & $0.8 \pm 0.2$ & 0.101 \\
\hline Timed up-and-go test (sec) & & & $14.0 \pm 4.7$ & & & $14.2 \pm 4.1$ & & & $13.2 \pm 5.8$ & 0.392 \\
\hline Handgrip strength $(\mathrm{kg})$ & & & $18.0 \pm 4.8$ & & & $18.0 \pm 4.5$ & & & $18.1 \pm 5.3$ & 0.926 \\
\hline Timed sit-to-stand test $(\mathrm{sec})^{* *}$ & & & $17.2 \pm 7.2$ & & & $15.0 \pm 5.1$ & & & $18.2 \pm 7.9$ & $0.048^{*}$ \\
\hline Mini-Mental State Examination score & & & $20.1 \pm 4.7$ & & & $19.5 \pm 4.8$ & & & $21.3 \pm 4.6$ & 0.095 \\
\hline EFT frailty score & & & $1.7 \pm 0.9$ & & & $1.8 \pm 0.9$ & & & $1.4 \pm 1.0$ & $0.026^{*}$ \\
\hline Orthostatic hypotension $\dagger$ & 29 & 31.9 & & 14 & 23.0 & & 15 & 50.0 & & $<0.01^{*}$ \\
\hline Orthostatic intolerance $\dagger$ & 22 & 25.0 & & 9 & 15.3 & & 13 & 44.8 & & $<0.01^{*}$ \\
\hline \multicolumn{11}{|l|}{ Heart rate } \\
\hline Supine & & & $72.8 \pm 11.4$ & & & $73.3 \pm 11.1$ & & & $71.6 \pm 12.0$ & 0.495 \\
\hline Standing immediate & & & $80.9 \pm 12.9$ & & & $81.4 \pm 12.2$ & & & $79.8 \pm 14.5$ & 0.592 \\
\hline Standing $1^{\text {st }} \min$ & & & $79.6 \pm 12.1$ & & & $79.6 \pm 10.4$ & & & $79.6 \pm 15.3$ & 0.992 \\
\hline Standing $3^{\text {rd }} \mathrm{min}$ & & & $79.7 \pm 12.7$ & & & $79.9 \pm 11.5$ & & & $79.3 \pm 15.2$ & 0.834 \\
\hline
\end{tabular}

SD: Standard deviation; EFT: Essential Frailty Toolset; ${ }^{*} \mathrm{p}<0.05$ statistically significant; ${ }^{\star}$ Test score represents the time needed to stand 5 times from a seated position; $\dagger$ Chi-squared test; Two independent sample T test were applied for all continuous data.

\begin{tabular}{|c|c|c|c|c|c|c|}
\hline \multicolumn{7}{|c|}{ Logistic regression analysis for pre } \\
\hline & \multicolumn{3}{|c|}{ Univariate analysis } & \multicolumn{3}{|c|}{ Multivariate analysis } \\
\hline & OR & $95 \% \mathrm{CI}$ & $p$ & OR & $95 \% \mathrm{CI}$ & $p$ \\
\hline Sarcopenia & 3.35 & $1.32-8.52$ & 0.011 & 7.80 & $1.77-34.45$ & 0.007 \\
\hline Age & 1.02 & $0.92-1.14$ & 0.636 & 1.09 & $0.94-1.25$ & 0.228 \\
\hline Sex & 1.85 & $0.64-5.32$ & 0.251 & 1.67 & $0.41-6.70$ & 1.670 \\
\hline Body mass index & 0.99 & $0.91-1.07$ & 0.822 & 1.08 & $0.95-1.23$ & 0.213 \\
\hline Vitamin-D & 0.99 & $0.96-1.02$ & 0.728 & 1.00 & $0.96-1.03$ & 0.983 \\
\hline
\end{tabular}

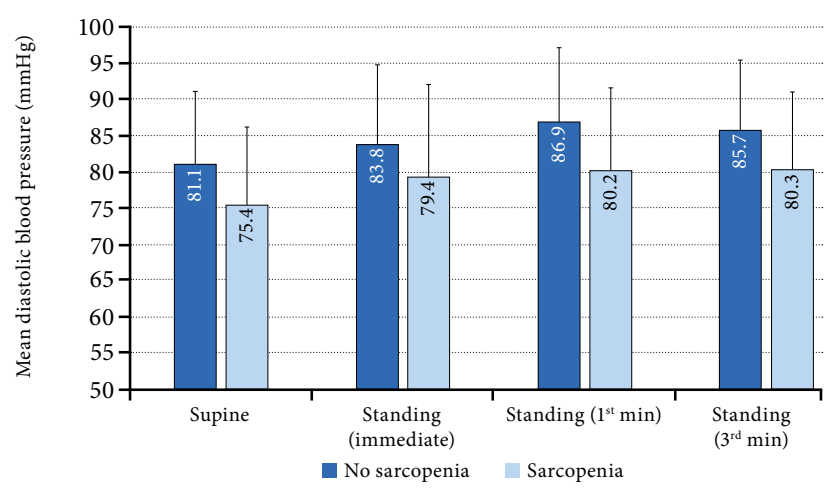

Figure 1. Supine and standing systolic blood pressure trends in elderly patients with and without sarcopenia.

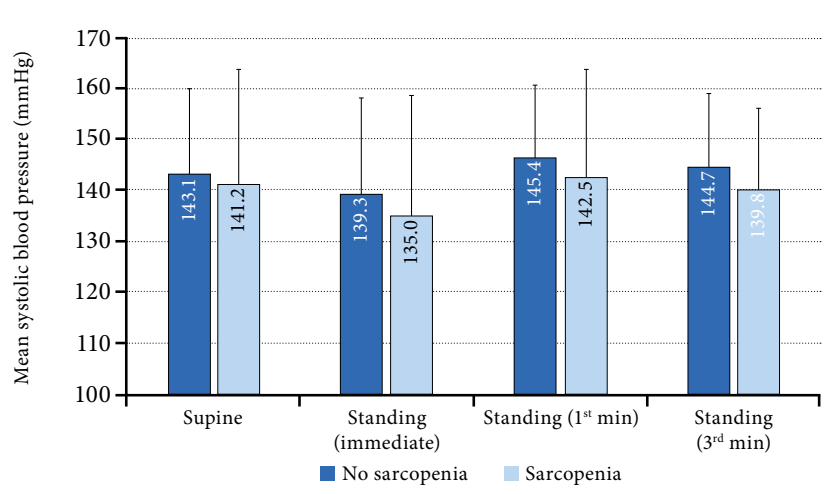

Figure 2. Supine and standing diastolic blood pressure trends in elderly patients with and without sarcopenia. 


\section{DISCUSSION}

In this study, we showed that there was a clear association between age-related sarcopenia and the frequency of $\mathrm{OH}$ even after adjusting for potential confounders. It seems that the risk of $\mathrm{OH}$ is increased in sarcopenic elderly compared to their non-sarcopenic counterparts.

It has long been recognized that elderly frail patients were prone to significant BP drops upon standing. Although the underlying mechanisms were not clearly established, these BP swings, particularly $\mathrm{OH}$, were attributed to the patients' general weakness, accompanying chronic conditions, low physical performance and deconditioning. Long-term follow-up studies consistently demonstrated that these patients had increased cardiovascular events, syncopal episodes and falls which led to significant injuries. ${ }^{[1]}$

On the other hand, the increased incidence of $\mathrm{OH}$ among frail elderly patients may be related to sarcopenia which is a condition that has a significant overlap with frailty. There is plenty of evidence showing that sarcopenia negatively affects balance, gait and imposes the risk of falls in the elderly as well. Although the definition of sarcopenia incorporates muscle loss along with diminished muscle strength or physical performance, it has a significant impact on the cardiovascular system. There are a number of studies showing that sarcopenia induces endothelial dysfunction, arterial stiffness and hypertension particularly in the long term. ${ }^{[7,8]}$ Moreover, a study conducted by Hashimoto et al. ${ }^{[12]}$ showed that significant systolic pressure variability was seen in patients with sarcopenia, a condition which is closely associated with falls and cardiovascular morbidity. Similarly, Kim et al. ${ }^{[13]}$ demonstrated a marked inverse relationship between within-visit BP variability and skeletal muscle mass in a large Korean population.

Apart from that, from a neurological point of view, from generation of a neural signal to muscle contraction, there are a lot of age-associated changes in nervous system which may eventually lead to muscle wasting. These changes also involve the autonomic nervous system which is directly related to BP regulation. ${ }^{[14]}$ Aagaard et al. ${ }^{[15]}$ showed that age-related loss of spinal motor neurons lead to the reduction in both the number and size of the muscle fibers (sarcopenia) and thus impairing muscle performance. The conduction velocities in the spinal cord also slow down as shown in transcranial magnetic stimulation studies. ${ }^{[16]}$ Similarly, Lauretani et al. ${ }^{[17]}$ examined the relationship of peroneal nerve conduction velocity and compound muscle action potentials with calf muscle mass and density which are the two complementary measures of sarcopenia. They showed that both nerve and muscle parameters declined with age. Taken together, a compromised nervous system not only induces sarcopenia, it may also play a role in BP disturbances to several stressors like standing.

One other possible mechanism by which $\mathrm{OH}$ occurs more frequently in patients with sarcopenia may be related to the weakness of the calf muscles which are responsible in the regulation of $\mathrm{BP}$ to upright postures. When an individual stands in a stationary position, there is an immediate fluid shift to the lower extremities due to the gravitational forces. In order to compensate the diminished flow to the heart, a number of compensatory mechanisms come into play, one of which is the activation of the physiologic skeletal muscle pump to induce muscle contraction in the legs and to provide pressure against veins and promote venous return to the heart. ${ }^{[18]}$ The importance of this mechanism is best appreciated in patients with spinal cord injuries where there is profound hypotension due to absence of muscle activity and sympathetic denervation. ${ }^{[19]}$ Further evidence comes from studies which apply functional electrical stimulation to the lower extremity muscles leading to improved venous return to the heart. ${ }^{[20]}$ Sarcopenia has similar autonomic dysfunctions at the neuromuscular junction level in which significant denervation, widening of synaptic clefts and alterations of acetylcholine receptors are observed. ${ }^{[21]}$ Therefore, muscle weakness of the lower extremities, particularly the calf muscles, may have a role for $\mathrm{OH}$ in these patients.

In our study, sarcopenic patients had lower vitamin D levels which may have certain implications on the outcome. First, vitamin D deficiency is closely related to vascular pathologies such as endothelial dysfunction, vascular stiffness and BP changes. ${ }^{[22]}$ Second, there are specific studies indicating that its deficiency may lead to $\mathrm{OH}$ as well. ${ }^{[23]}$ Recently, a study led by Soysal et al. ${ }^{[24]}$ found that vitamin D deficiency may increase the risk of $\mathrm{OH}$ in the elderly. Although vitamin D may have certain effects on the postural BP changes, we cannot definitely propose this finding as a possible mechanism since in multivariate analysis sarcopenia was the only independent predictor of $\mathrm{OH}$ development. Nevertheless, this is an interesting finding that deserves further investigation.

Moreover, since this was a proof of concept study, we believe that a greater understanding of the complex mechanisms underlying the association between sarcopenia and $\mathrm{OH}$ should be explored which will 
hopefully facilitate the development of beneficial interventions.

There were some limitations in our study. First, it was a single-center study with a relatively small sample size. Second, since we only investigated elderly patients with age-related sarcopenia, our findings cannot be extrapolated to other forms of sarcopenia or the sarcopenia seen in younger patients.

In conclusion, in our study, $\mathrm{OH}$ was more common in elderly patients with sarcopenia compared to non-sarcopenic elderly. This may in part explain the increased incidence of falls and help identification of risky elderly patients for orthostatic BP drops. However, the underlying mechanisms should be explored further.

Acknowledgments

We would like to thank all nurses working in the cardiology outpatient department for their help during patient recruitment.

\section{Declaration of conflicting interests}

The authors declared no conflicts of interest with respect to the authorship and/or publication of this article.

\section{Funding}

The authors received no financial support for the research and/or authorship of this article.

\section{REFERENCES}

1. Cruz-Jentoft AJ, Baeyens JP, Bauer JM, Boirie Y, Cederholm T, Landi F, et al. Sarcopenia: European consensus on definition and diagnosis: Report of the European Working Group on Sarcopenia in Older People. Age Ageing 2010;39:412-23.

2. Gadelha AB, Vainshelboim B, Ferreira AP, Neri SGR, Bottaro M, Lima RM. Stages of sarcopenia and the incidence of falls in older women: A prospective study. Arch Gerontol Geriatr 2018;79:151-7.

3. Öztürk ZA, Türkbeyler İH, Abiyev A, Kul S, Edizer B, Yakaryılmaz FD, et al. Health-related quality of life and fall risk associated with age-related body composition changes; sarcopenia, obesity and sarcopenic obesity. Intern Med J 2018;48:973-81.

4. Hida T, Harada A, Imagama S, Ishiguro N. Managing sarcopenia and its related-fractures to improve quality of life in geriatric populations. Aging Dis 2013;5:226-37.

5. Palus S, Springer JI, Doehner W, von Haehling S, Anker M, Anker SD, et al. Models of sarcopenia: Short review. Int J Cardiol 2017;238:19-21.

6. Janssen I. Influence of sarcopenia on the development of physical disability: the Cardiovascular Health Study. J Am Geriatr Soc 2006;54:56-62.

7. Kohara K, Okada Y, Ochi M, Ohara M, Nagai T, Tabara $\mathrm{Y}$, et al. Muscle mass decline, arterial stiffness, white matter hyperintensity, and cognitive impairment: Japan Shimanami Health Promoting Program study. J Cachexia Sarcopenia Muscle 2017;8:557-66.
8. Dos Santos MR, Saitoh M, Ebner N, Valentova M, Konishi M, Ishida J, et al. Sarcopenia and Endothelial Function in Patients With Chronic Heart Failure: Results From the Studies Investigating Comorbidities Aggravating Heart Failure (SICA-HF). J Am Med Dir Assoc 2017;18:240-5.

9. Afilalo J, Lauck S, Kim DH, Lefèvre T, Piazza N, Lachapelle $\mathrm{K}$, et al. Frailty in older adults undergoing aortic valve replacement: The FRAILTY-AVR study. J Am Coll Cardiol 2017;70:689-700.

10. Consensus statement on the definition of orthostatic hypotension, pure autonomic failure, and multiple system atrophy. The Consensus Committee of the American Autonomic Society and the American Academy of Neurology. Neurology 1996;46:1470.

11. O'Connell MD, Savva GM, Fan CW, Kenny RA. Orthostatic hypotension, orthostatic intolerance and frailty: The Irish Longitudinal Study on Aging-TILDA. Arch Gerontol Geriatr 2015;60:507-13.

12. Hashimoto Y, Kaji A, Sakai R, Hamaguchi M, Okada H, Ushigome E, et al. Sarcopenia is associated with blood pressure variability in older patients with type 2 diabetes: A cross-sectional study of the KAMOGAWA-DM cohort study. Geriatr Gerontol Int 2018;18:1345-9.

13. Kim KI, Kang MG, Yoon SJ, Choi JY, Kim SW, Kim $\mathrm{CH}$. Relationship between within-visit blood pressure variability and skeletal muscle mass. J Nutr Health Aging 2019;23:79-83.

14. Kwon YN, Yoon SS. Sarcopenia: Neurological point of view. J Bone Metab 2017;24:83-9.

15. Aagaard P, Suetta C, Caserotti P, Magnusson SP, Kjaer $M$. Role of the nervous system in sarcopenia and muscle atrophy with aging: strength training as a countermeasure. Scand J Med Sci Sports 2010;20:49-64.

16. SaleMV,SemmlerJG. Age-related differencesin corticospinal control during functional isometric contractions in left and right hands. J Appl Physiol 2005;99:1483-93.

17. Lauretani F, Bandinelli S, Bartali B, Di Iorio A, Giacomini V, Corsi AM, et al. Axonal degeneration affects muscle density in older men and women. Neurobiol Aging 2006;27:1145-54.

18. Ricci F, De Caterina R, Fedorowski A. Orthostatic Hypotension: Epidemiology, Prognosis, and Treatment. J Am Coll Cardiol 2015;66:848-60.

19. Wecht JM, Bauman WA. Implication of altered autonomic control for orthostatic tolerance in SCI. Auton Neurosci 2018;209:51-8.

20. Faghri PD, Yount J. Electrically induced and voluntary activation of physiologic muscle pump: a comparison between spinal cord-injured and able-bodied individuals. Clin Rehabil 2002;16:878-85.

21. Wokke JH, Jennekens FG, van den Oord CJ, Veldman H, Smit LM, Leppink GJ. Morphological changes in the human end plate with age. J Neurol Sci 1990;95:291-310.

22. Al Mheid I, Patel R, Murrow J, Morris A, Rahman A, Fike $\mathrm{L}$, et al. Vitamin D status is associated with arterial stiffness and vascular dysfunction in healthy humans. J Am Coll Cardiol 2011;58:186-92.

23. Mann MC, Exner DV, Hemmelgarn BR, Sola DY, Turin TC, Ellis $\mathrm{L}$, et al. Vitamin D levels are associated with cardiac autonomic activity in healthy humans. Nutrients 2013;5:2114-27.

24. Soysal P, Yay A, Isik AT. Does vitamin D deficiency increase orthostatic hypotension risk in the elderly patients? Arch Gerontol Geriatr 2014;59:74-7. 